(2) Open Access Full Text Article

REVIEW

\title{
Darunavir-cobicistat-emtricitabine-tenofovir alafenamide: safety and efficacy of a protease inhibitor in the modern era
}

This article was published in the following Dove Press journal:

Drug Design, Development and Therapy

\author{
Nicola Squillace' \\ Giorgio Bozzi ${ }^{2}$ \\ Elisa Colella' \\ Andrea Gori ${ }^{2}$ \\ Alessandra Bandera' \\ 'Infectious Diseases Unit, Azienda \\ Socio Sanitaria Territoriale di Monza, \\ San Gerardo Hospital, University \\ of Milano-Bicocca, Monza, Italy; \\ ${ }^{2}$ Infectious Disease Unit, Department \\ of Internal Medicine, Fondazione \\ IRCCS Ca'Granda, Ospedale \\ Maggiore Policlinico, Department of \\ Pathophysiology and Transplantation, \\ University of Milano, Milan, Italy
}

\begin{abstract}
A fixed-dose combination consisting of darunavir (Drv), cobicistat (Cobi), emtricitabine (2', $3^{\prime}$-dideoxy-5-fluoro-3'-thiacytidine [FTC]), and tenofovir alafenamide (Taf) has been recently approved by the European Medicines Agency for the treatment of HIV infection, and is the first ever protease-inhibitor-based single-tablet regimen. This article provides a detailed description of its pharmacokinetic, efficacy, and safety profile. The pharmacokinetics of single compounds were analyzed, with a special focus on contrasts between Drv/Cobi and Drv/ritonavir (Rtv). When comparing Cobi and Rtv, multiple interactions must be taken into account: in comparison to Rtv, Cobi is a more selective CYP3A4 inhibitor and has no clinical effect on other isoenzymes inhibited by Rtv (eg, 2C8 and 2C9). Moreover, unlike Cobi, Rtv shows in vivo induction activity on some CYP isoenzymes (eg, 1A2, 2C19, 2C8, 2C9, and 2B6), glucuronyltransferases (eg, UGT1A4), and Pgp. Drv-Cobi-FTC-Taf has recently been demonstrated to be of equal efficacy to Drv-Rtv and other protease inhibitors in both experienced (EMERALD study) and naïve (AMBER study) patients. Moreover, kidney and bone safety profiles have been shown to be good, as has central nervous system tolerance. Total cholesterol:low-density-lipoprotein cholesterol and total cholesterol:high-density-lipoprotein cholesterol ratios are generally high in Drv-Cobi-FTC-Taf vs Rtv-Drv-FTC + tenofovir disoproxil fumarate. An unlikely role of Drv in influencing cardiovascular risk in HIV infection has also been reported. Kidney safety profile is influenced by Cobi, with an increase in creatinine plasma concentration of $0.05-0.1 \mathrm{mg} / \mathrm{dL}$ and a parallel glomerular filtration-rate reduction of $10 \mathrm{~mL} / \mathrm{min}$ within the first 4 weeks after Cobi introduction, which remains stable during treatment. Bone and central nervous system safety profiles were found to be good in randomized clinical trials of both experienced and naïve patients. The efficacy and safety of Drv/Cobi/FTC/ Taf are comparable to other drug regimens recommended for HIV treatment.
\end{abstract}

Keywords: protease inhibitors, darunavir/cobicistat, emtricitabine/tenofovir alafenamide, HIV

\section{Introduction}

The use of protease inhibitors (PIs) as a component of combination antiretroviral therapy (ART) has radically improved the prognosis of HIV infection, leading to significant and durable control with immunorestoration and increasing life expectancy. ${ }^{1}$ In the first years of introduction, however, high efficacy was counterbalanced by a large burden of adverse events, such as lipodystrophy, dyslipidemia, and insulin resistance. ${ }^{2}$ Atazanavir (Atv) was the first PI prescribed once daily, followed by darunavir (Drv) which proved to be as efficacious as other PIs, but with a better lipid and metabolic profile and a lower rate of lipodystrophy. ${ }^{3,4}$ Recently developed,
Correspondence: Nicola Squillace Infectious Diseases Unit, Azienda Socio Sanitaria Territoriale di Monza, San Gerardo Hospital, University of MilanoBicocca, 33 Via Pergolesi, Monza 20900, Italy

Tel +390392339588

Fax +390392339327

Email n.squillace@asst-monza.it 
tenofovir alafenamide (Taf) has been shown to be equally effective and have lower toxicity than tenofovir disoproxil fumarate (TDF). ${ }^{5-7}$

The introduction of single-tablet regimens (STRs) has changed the scenario in the management of HIV infection, significantly increasing adherence and quality of life of people living with HIV. ${ }^{8}$ Until last year, STRs were available only for nonnucleoside reverse-transcriptase inhibitors and integrase inhibitors. The newly approved one-pill combination of Drv, cobicistat (Cobi), emtricitabine (2',3'-dideoxy-5-fluoro-3'-thiacytidine [FTC]), and tenofovir alafenamide (Taf), being PI-based, thus represents a novelty in HIV therapy. The aim of this review was to evaluate the pharmacokinetics, efficacy, and safety of DrvCobi-FTC-Taf.

\section{Drv-Cobi-FTC-Taf pharmacokinetics Taf}

After intestinal absorption, Taf enters hepatocytes by passive diffusion, facilitated by organic anion-transporting polypeptides $1 \mathrm{~B} 1$ and $1 \mathrm{~B} 3$. It is then metabolized by carboxylesterase 1 to form tenofovir (Tfv), which phosphorylates to Tfv diphosphate, its active metabolite..$^{9,10}$ Taf is more stable than TDF in plasma: in vitro, the plasma halflife of Taf is 30-90 minutes compared to 0.4 minutes for TDF, allowing for efficient uptake by hepatocytes. ${ }^{11,12} \mathrm{Tfv}$ is eliminated through the kidneys by both active proximal tubule secretion and passive glomerular filtration: lowering the administered Tfv equivalents decreases kidney exposure. Incubation of primary human hepatocytes with Taf in vitro shows high intracellular concentrations of Tfv diphosphate, approximately five and 120 times higher than concentrations observed with TDF and Tfv, respectively. Tfv diphosphate has been demonstrated to be a potent inhibitor of HBV replication. ${ }^{10,13}$ In severely hepatically impaired (Child-Pugh C) patients uninfected with HIV treated with Taf $25 \mathrm{mg}$, Tfv exposure has been reported to be only modestly lower compared to healthy controls with normal hepatic function. ${ }^{14}$ In patients with severe renal impairment receiving treatment with Taf $25 \mathrm{mg}$, plasma levels of Tfv were lower than those historically seen in pharmacokinetic studies using TDF in antiretroviral regimens for HIV-infected patients with normal renal function, albeit higher than in matched healthy subjects. ${ }^{14}$ A study on the effect of food on Taf pharmacokinetics showed that Taf exposure decreased in fasting conditions. ${ }^{15}$ However, no significant trend in exposure response/safety regarding Taf exposure was found in fasting or fed conditions. ${ }^{11}$

\section{Drv-Cobi}

Like most PIs, Drv is metabolized extensively through CYP3A4 in the liver and the gut. ${ }^{16}$ Once-daily administration and lower doses can thus be achieved by coadministering a pharmacokinetic enhancer. Until recently, low-dose ritonavir (Rtv; $100 \mathrm{mg}$ once or twice daily), a strong CYP3A4 and Pgp inhibitor, was the staple pharmacokinetic booster available. ${ }^{17}$ However, this enhancement is not limited to antiretroviral drugs, but affects other drugs metabolized through CYP, with risk of side effects. In order to overcome this issue, a new antiretroviral drug booster, Cobi, was developed from the Rtv molecule. Despite being similar to Rtv in many aspects, Cobi inhibits CYP isoenzymes more selectively and does not cause induction effects on glucuronidation or CYP. ${ }^{18}$ Furthermore, Cobi has high aqueous solubility and can be readily coformulated with other agents. It is currently used in fixed-dose combinations (FDCs) to enhance plasma concentrations of elvitegravir, Atv, and Drv. Importantly, while Rtv has activity against HIV, possibly leading to the development of drug-resistance mutations to PIs in cases of suboptimal regimens, Cobi is devoid of intrinsic activity against HIV replication. ${ }^{19,20}$ Recently, Drv $800 \mathrm{mg}$ and Cobi $150 \mathrm{mg}$ have become available in an FDC (Rezolsta; Johnson and Johnson, New Brunswick, NJ, USA).

Drv achieves peak plasma concentration $\left(\mathrm{C}_{\max }\right)$ in 3-4.5 hours after oral administration of Drv-Cobi 800/150 mg. Administration with food significantly increases Drv absorption, raising $\mathrm{C}_{\max }$ more than double and Drv area under the curve (AUC) up to 1.7-fold (Cobi pharmacokinetics are unaffected). ${ }^{21,22}$ Cobi binds to plasma proteins at up to $98 \%$, and is mostly eliminated through feces $(86 \%) .{ }^{23}$ In a study involving healthy volunteers, apparent volume of distribution was 269 and $158 \mathrm{~L}$ for Cobi 100 and $200 \mathrm{mg} /$ day, respectively. ${ }^{24}$ Cobi has a short median plasma half-life (3-4 hours). ${ }^{23}$ In a phase 1, open-label, randomized clinical trial comparing the bioavailability of two distinct Drv-Cobi $800 / 150 \mathrm{mg}$ oncedaily coformulations and Drv-Rtv 800/100 mg once daily, Drv AUC 24 hours and $\mathrm{C}_{\max }$ with either enhancer were found to be comparable. ${ }^{25}$ In a phase IIIB study of $60 \mathrm{HIV}$-infected patients, the pharmacokinetic profile of Drv was analyzed by administering Drv $800 \mathrm{mg}$ and Cobi $150 \mathrm{mg}$ (as single agents) with any nucleoside reverse-transcriptase-inhibitor backbone (mostly TDF-based). ${ }^{26}$ Mean Drv AUC, $\mathrm{C}_{\max }$, and $\mathrm{C}_{\text {trough }}$ values $\pm \mathrm{SD}$ were $81,646 \pm 26,322 \mathrm{ng} \cdot \mathrm{h} / \mathrm{mL}, 7,663 \pm 1,920 \mathrm{ng} / \mathrm{mL}$, and $1,311 \pm 969 \mathrm{ng} / \mathrm{mL}$, respectively, comparable to results observed in healthy volunteers..$^{20,25,27,28}$ Geometric mean values 24 hours after the observed dose for Cobi-boosted Drv were reported to be $43 \mathrm{ng} / \mathrm{mL}$ in saliva and $11,878 \mathrm{ng} / \mathrm{mL}$ in 
urine. Concentration decay in saliva/urine was found to mirror plasma concentrations. ${ }^{29}$ Cobi and Rtv produce comparatively effective Drv concentrations in cerebrospinal fluid. ${ }^{30}$

\section{Drug-drug interactions with Drv-Cobi}

As mentioned, Cobi functions primarily as a pharmacokinetic enhancer by means of CYP3A4 inhibition. However, other enzymes and transporters are weakly inhibited by Cobi (see Figure 1). ${ }^{20,21,27}$ In comparison to Rtv, Cobi is a more selective CYP3A4 inhibitor, as it is devoid of effects on other isoenzymes. It also lacks the induction activity of Rtv on several enzymes and transporters, including CYP isoenzymes, Pgp, and glucuronyltransferases. ${ }^{15,31,32}$

Table 1 summarizes the main differences between Rtv and Cobi. ${ }^{31}$ Coadministration of Cobi and Pgp substrates (ie, digoxin), may increase the latter drug's plasma levels, possibly leading to adverse events. ${ }^{33}$ Conversely, coadministered drugs that inhibit/induce CYP3A4 will affect Cobi plasma concentrations. Cobi concentrations are increased by coadministration with other inhibitors, increasing the risk of toxicity, while in contrast CYP3A inducers may lower Cobi concentrations, possibly leading to the emergence of drug-resistance mutations and virological failure. Importantly, as some nonnucleoside reverse-transcriptase inhibitors, namely etravirine, efavirenz, or nevirapine (but not rilpivirine), can be strong inducers, their coadministration with Cobi is contraindicated. ${ }^{34}$ Relevant discrepancies between Cobi and Rtv have been reported for interactions with rifabutin ${ }^{35,36}$ and warfarin. ${ }^{37}$ There are no available data on the usage of Cobi as a pharmacokinetic enhancer for PIs other than atazanavir or Drv. Furthermore, the Drv-Cobi

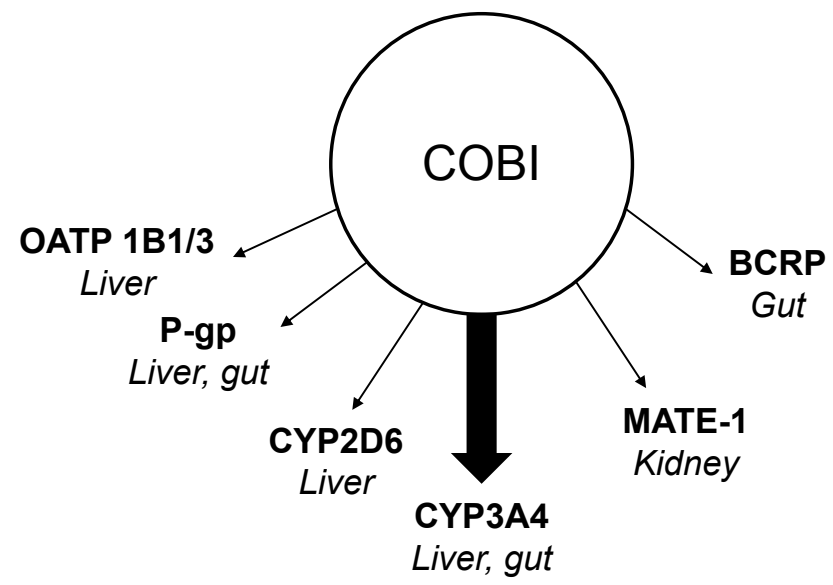

Figure I Activity of cobicistat (Cobi).

Abbreviations: $\mathrm{BRCP}$, breast cancer resistance protein; $\mathrm{COBI}$, cobicistat; CYP2D6, Cytochrome P450 2D6; CYP3A4, Cytochrome P450 3A4; MATE-I, Multidrug and toxin extrusion protein-I; OATP, organic-anion-transporting polypeptide; P-gp, P-glycoprotein. combination is not potent enough to boost the activity of other coadministered drugs, such as elvitegravir, meaning that coadministration might pose a risk of suboptimal plasma levels, possibly leading to virological failure..$^{35,38}$

\section{Drv-Cobi-FTC-Taf fixed-dose combination pharmacokinetics phase I studies}

The phase I trial NCT02578550 confirmed the bioequivalence of FDC Drv-Cobi-FTC-Taf relative to the separate agents (Drv $800 \mathrm{mg}$ tablet formulation and FTC-Taf 200/10 mg FDC) in the presence of $150 \mathrm{mg}$ Cobi under fed conditions in healthy participants (see Table 2). The impact of food on the bioavailability of coformulated Drv-Cobi-FTC-Taf was tested in the phase I trial NCT02475135. ${ }^{39}$ Food effects with Drv were observed only after administration of Drv-CobiFTC-Taf. It is thus recommended that Drv-Cobi-FTC-Taf be taken with food, which is also the recommendation in the ongoing phase III trials. ${ }^{40}$

\section{Efficacy: phase II and III studies}

In an exploratory phase II trial (ClinicalTrials.gov NCT01565850; GS-US-299-0102) ${ }^{41}$ in 153 treatment-naïve adults with estimated glomerular filtration rate $(\mathrm{GFR}) \geq 70$ $\mathrm{mL} / \mathrm{min}$, the virological efficacy of the Drv-Cobi-FTC-Taf regimen was compared to a regimen containing Drv and Cobi combined with FTC and TDF with 2:1 double-blinded randomization (see Table 2). ${ }^{42}$ At week 24, viral suppression (HIV1 RNA $<50$ copies/mL) rates were similar $(74.8 \%$ for Taf vs 74.0\% for TDF; US Food and Drug Administration [FDA] snapshot analysis, primary end point, with a $12 \%$ margin for noninferiority). At week 48, however, rates were $76.7 \%$ for the Taf-containing STR vs $84.0 \%$ for the TDF-containing regimen. This difference appeared to be driven by a higher rate of discontinuations in the Taf (6.8\%) vs TDF arm (2\%). On the other hand, significant renal and bone safety improvements were observed in the Taf arm. Of note, no resistance to any compound was observed. A single-tablet, once-daily regimen of Drv $800 \mathrm{mg}-\mathrm{Cobi}$ $150 \mathrm{mg}-$ FTC $200 \mathrm{mg}$-Taf $10 \mathrm{mg}$ is currently under investigation in two international, randomized, phase III studies: EMERALD (ClinicalTrials.gov NCT02269917) and AMBER (NCT02431247; see Table 2). ${ }^{43,44}$

EMERALD was a randomized, active-controlled, open-label, international, multicenter trial carried out in nine countries in North America and Europe that began on March 30, 2015. ${ }^{45}$ The aim of the study was to evaluate safety and efficacy of single-tablet Drv-Cobi-FTC-Taf as a potential switch option 
Table I Principal differences in drug interactions between booster dosing of ritonavir (Rtv) and cobicistat (Cobi)

\begin{tabular}{|l|l|l|}
\hline Drug & Rtv & Cobi \\
\hline Etravirine (Etv) & No interactions & $\downarrow \downarrow \downarrow$ Etv* \\
\hline Nevirapine (Nev) & No interactions & $\downarrow \downarrow \downarrow$ Nev* \\
\hline Efavirenz (Efv) & $\uparrow$ Efv, $\uparrow$ Rtv & $\downarrow \downarrow \downarrow$ Cobi* \\
\hline Rifabutin & $\uparrow$ Rifabutin & $\downarrow \downarrow$ Cobi \\
\hline Olanzapine & $\downarrow$ Olanzapine & No interactions \\
\hline Sertraline & $\downarrow$ Sertraline & $\uparrow$ Sertraline \\
\hline Carbamazepine & $\uparrow$ Carbamazepine & $\downarrow \downarrow \downarrow$ Cobi* \\
\hline Acenocumarol & $\uparrow$ Acenocumarol & No interactions \\
\hline Propofol & $\downarrow$ Propofol & No interactions \\
\hline Lamotrigine & $\downarrow$ Lamotrigine & No interactions \\
\hline Valproate & $\downarrow$ Valproate & No interactions \\
\hline Gliclazide & $\downarrow$ Gliclazide & No interactions \\
\hline Metformin & No interactions & $\uparrow$ Metformin exposure \\
\hline Mycophenolic acid & Variable interactions: $\downarrow \uparrow$ & No interactions \\
\hline Gemfibrozil & $\downarrow$ Gemfibrozil & No interactions \\
\hline Pitavastatin & No interactions & Pitavastatin \\
\hline
\end{tabular}

Notes: $\uparrow$, Potential increase in drug exposure; $\uparrow \uparrow$, moderate increase in drug exposure; $\uparrow \uparrow \uparrow$, significant increase in drug exposure; $\downarrow$, potential decrease in drug exposure; $\downarrow \downarrow$, moderate decrease in drug exposure; $\downarrow \downarrow \downarrow$, significant decrease in drug exposure. Reference www.hiv-druginteractions.org, *contraindicated.

Abbreviations: Etv, etravirine; Nev, nevirapine; Efv, efavirenz; Rtv, ritonavir; Cobi, cobicistat.

for the treatment of HIV1 infection in adults with viral suppression. Treatment-experienced and virologically suppressed (viral load $<50$ copies/mL for $\geq 2$ months) HIV1-infected adults were eligible. One viral load of 50-200 copies $/ \mathrm{mL}$ was allowed within 12 months before screening, and patients with a history of virological failure on regimens other than Drvcontaining were allowed. Patients were randomly assigned (2:1) to switch to the open-label study regimen or continue the control regimen. The primary outcome was the proportion of participants with virological rebound (either confirmation of viral load $\geq 50$ copies $/ \mathrm{mL}$ or premature discontinuation, with last viral load $\geq 50$ copies $/ \mathrm{mL}$ ). Noninferiority was tested (4\% margin) vs the control regimen in the intention-to-treat population. Of 1,141 total patients (763 and 378 in the study and control groups, respectively), $58 \%$ had previously received five or more antiretrovirals, including screening drugs, and $15 \%$ had experienced previous non-Drv virological failure. The study regimen was found to be noninferior for virological rebound ( $2.5 \%$ patients vs $2.1 \%$ in the study and control groups, respectively; difference $0.4 \%, 95 \% \mathrm{CI}-1.5-2.2 ; P<0.0001$ ). No accumulation of drug resistance was observed. The proportion of adverse event-related discontinuations ( $1 \%$ in the study group vs $1 \%$ in the control group) and grade $3-4$ adverse events ( $7 \%$ vs $8 \%$, respectively) was similar between groups. A small, statistically significant $(0.2 \pm 1.1$ vs $0.1 \pm 1.1, P=0.010)$ difference between groups in change from baseline in total:high-density lipoprotein (HDL) cholesterol ratio was reported; however, this difference was not deemed to be clinically relevant. Only one serious adverse event (a case of pancreatitis) was reported to be possibly related to the study regimen.

The purpose of the AMBER study ${ }^{46}$ is to demonstrate efficacy noninferiority of a Drv-Cobi-FTC-Taf FDC tablet vs Drv-Cobi FDC coadministered with FTC-TDF in 725 HIV1-infected, ART-naïve adult participants in the context of a multicenter, randomized, double-blind, active-controlled study beginning July 6,2015 . The primary outcome of the study is the percentage of participants obtaining HIV RNA $<50$ copies/mL defined by the FDA snapshot approach (time frame: week 48). Unpublished results at week 48 showed the FDC to be noninferior to the combination of DrvCobi and Taf-FTC in terms of efficacy (with $91.4 \%$ vs $88.4 \%$ patients achieving virological suppression, respectively). ${ }^{46}$ Interestingly, neither arm developed Drv, primary PI or TDFTaf Resistance Associated Mutations (RAMs). One patient in the Drv-Cobi-Ftc-Taf arm, however, developed M184I/V, which confers high-level in vitro resistance to lamivudine and FTC while increasing susceptibility to TDF ${ }^{47}$ Few serious adverse events or adverse event-related discontinuations were reported: diarrhea and rash were the most commonly reported adverse events in both arms. 


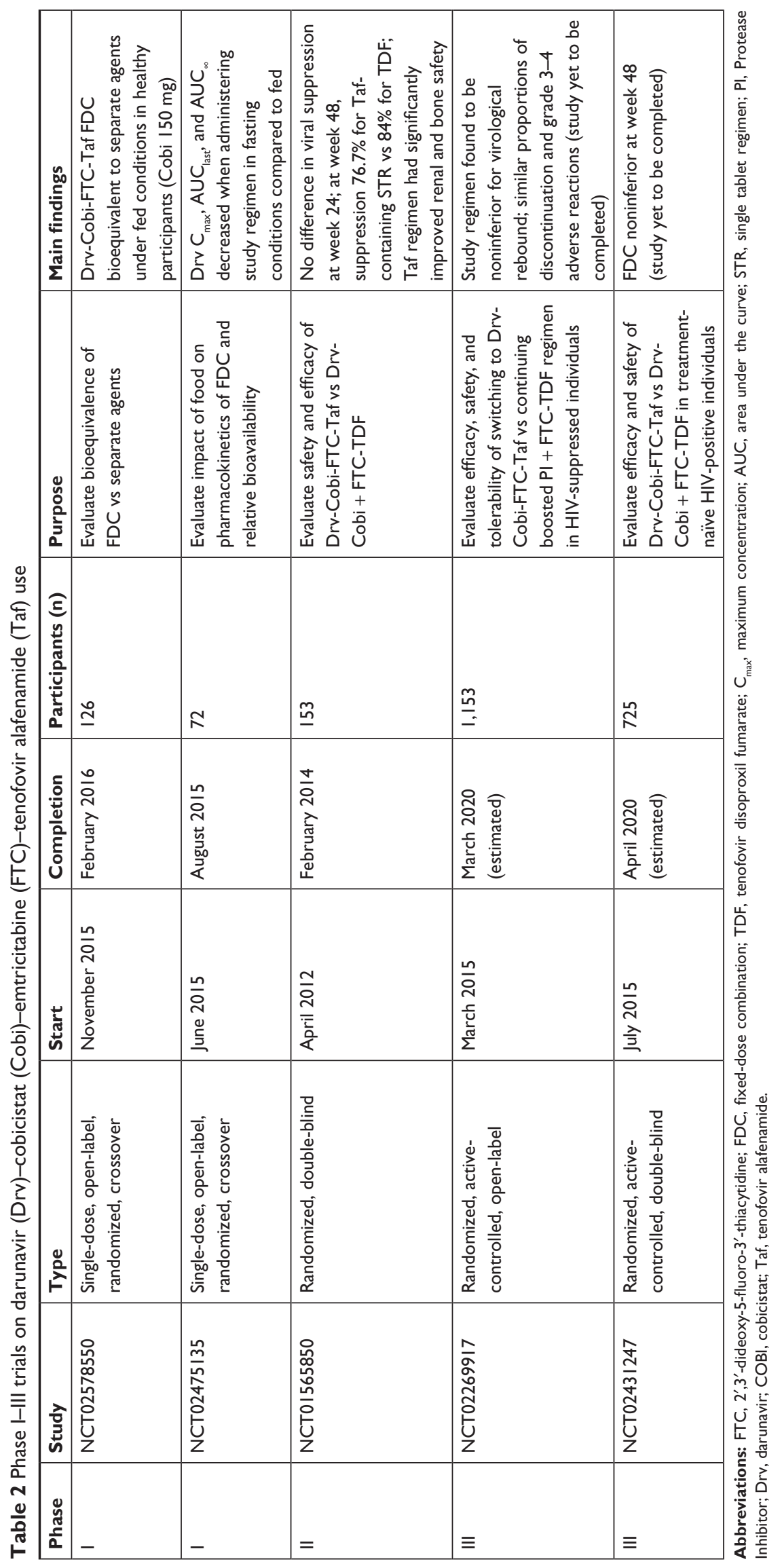




\section{Drv-Cobi safety}

Available data regarding Drv-Cobi 800/150 mg once-daily combination safety and tolerability reveal mild adverse events, due to gastrointestinal disturbances (diarrhea, nausea, flatulence) and rash or other skin reaction. ${ }^{26}$ During the Drv clinical development program, severe skin reactions were occasionally described, while in the postmarketing phase grade acute skin reactions were at times reported. Caution must be taken with patients with past hypersensitivity reactions during sulfonamide use, because of the reported higher probability of developing the same adverse event with Drv. ${ }^{21,26}$

The updated unpublished AMBER study reported the tolerability profile of Drv-Cobi-FTC-Taf at 48 weeks to be good (randomized double-blind phase: Drv-Cobi-FTC-Taf matched with TDF-FTC + Drv-Cobi placebo vs TDF-FTC + Drv-Cobi matched with Drv-Cobi-FTC-Taf placebo), with no discontinuations due to renal, bone, or central nervous system adverse events. ${ }^{46}$ Cobi inhibits human renal transporters (see Figure 1).$^{48}$ Because of those inhibitions in urinary creatinine excretion, an increase in creatinine plasma concentration of $0.05-0.1 \mathrm{mg} / \mathrm{dL}$ and a parallel GFR reduction of $10 \mathrm{~mL} / \mathrm{min}$ are expected within the first 4 weeks after Cobi introduction, with stabilization occurring during treatment and resolving with Cobi discontinuation, with no effect on actual clearance. ${ }^{49,50}$ Drv-Cobi was also studied in patients with no severe renal impairment in the GS-US-236-118 Study, which showed stable estimated GFR (eGFR; measured by cystatin C) at week $96 .{ }^{51}$ The metabolic profile of DrvCobi-FTC-Taf observed at 48 weeks in the EMERALD ${ }^{45}$ and AMBER studies revealed significant increases in total cholesterol, low-density lipoprotein (LDL) cholesterol, and total cholesterol:HDL-cholesterol ratio, with no significant increase in lipid-lowering therapy prescription in the study arm. These results were partially attributable to the process of comparing Taf vs TDF in both studies, given the former's relative lack of lipid-lowering effect. ${ }^{52}$

The cardiovascular safety of Drv has also been studied through postmarketing analysis comparing its effects on endothelial cell function with those of other PIs. Drv has been shown to have minimal or no effect on induction of endothelial oxidative stress and inflammation (ie, phosphorylation of p65/RelA-NFKB) compared to atazanavir and lopinavir. ${ }^{53}$ Secretion of soluble ICAM or VCAM has been shown not to be significantly altered by Drv or Drv-Rtv. ${ }^{53}$ Similar results were observed regarding senescence markers, such as senescence-associated $\beta$-galactosidase activation and overexpression of phospho-p53, p16 $6^{\mathrm{INK} 4}, \mathrm{p} 21^{\mathrm{WAF} 1}$, and prelamin $\mathrm{A} . .^{53}$ Instead, interestingly, an intermediate effect of Atv-Rtv and a strong effect of lopinavir-Rtv on all these inflammation and endothelial activation markers has been demonstrated.$^{53}$ Evaluation of cardiovascular risk associated with Drv-Rtv has produced conflicting results, both in randomized clinical trials and observational studies. In the MONARCH trial, ${ }^{54}$ no effect on endothelial function (measured by flow-mediated dilation) was observed when switching from a standard ART regimen to Drv/Rtv monotherapy or to Drv-Rtv + a combination of nucleoside reverse-transcriptase inhibitors. Data from the DAD study showed a markedly increased incidence of cardiovascular events associated with Drv-Rtv in a cohort of 35,711 patients with a median follow-up of 7 years. ${ }^{55}$

Furthermore, recent unpublished data based on CVD events in postmarketing pharmacovigilance databases conducted on 5,721 patients enrolled in 19 Janssen-sponsored clinical trials did not indicate an increased risk of CVD events with Drv-Rtv use in treatment duration up to 6 years, although a significantly higher rate of cardiovascular events in patients receiving Drv 600-Rtv $100 \mathrm{mg}$ twice daily vs Drv 800-Rtv $100 \mathrm{mg}$ once daily was observed. ${ }^{56}$ Data on cardiovascular events in patients on Drv-Cobi-FTC-Taf are scarce: neither the EMERALD nor the AMBER study reported a significant incidence of such adverse events..$^{45,46}$ Further studies are needed to evaluate the long term safety of this compound and to ascertain the unlikely association of Drv with cardiovascular events. Few studies have directly addressed Drv and Cobi bone toxicity/safety. It is possible to approximate Drv and Cobi bone safety profiles by examining the results of the NEAT trial, which compared Drv-Rtv + raltegravir to Drv-Rtv + TDF-FTC at 48 weeks: differences in bone-mineral density (BMD) changes were more pronounced in the TDF-containing regimen, where significant increases in serum markers of bone turnover were observed. ${ }^{57,58}$

A recent study evaluated impact on bone by Drv-CobiFTC and TDF/Taf. At week 48, a better BMD profile was reported in the Taf group: compared to the TDF group, more patients had an increase in BMD at the hip or spine $(5 \%$ and $11 \%$ for Taf vs 0 and $2 \%$ for TDF, respectively), and fewer patients had decreased BMD at the hip or spine (18\% and $33 \%$ for Taf vs $62 \%$ and $55 \%$ for TDF, respectively). Interestingly, an AIDS Clinical Trials Group (ACTG) A5257 substudy found similar BMD loss among regimens with different PIs, such as Atv-Rtv and Drv-Rtv, but lower in patients receiving raltegravir. Of note, all subjects in the two PI arms received TDF, and because Rtv coadministration increases $\mathrm{Tfv}$ concentrations by approximately $30 \%$, 
some of the effects described might have to be related to enhanced TDF exposition, rather than to a direct effect of PIs on bone. ${ }^{59,60}$ Conversely, data from ACTG A5224s showed a similar PI effect (Atv-Rtv) with either TDF-FTC or abacavir-lamivudine. ${ }^{61}$

The recently published EMERALD study had a boneinvestigation substudy investigating the efficacy and safety of switching to a single-tablet Drv-Cobi-FTC-Taf regimen vs continuing a regimen of boosted PI plus TDF-FTC. The substudy reported a BMD increase at the hip, lumbar spine, and femoral neck at week 48 in the study group $(P<0.0001$ for ANCOVA within-treatment comparison at hip and lumbar spine and $P=0.029$ for femoral neck). Conversely, in the control group, at week 48 hip BMD was stable, whereas lumbar spine and femoral neck BMD had decreased $(P=0.78$ for ANCOVA within-treatment comparison at the hip, $P=0.98$ for lumbar spine, and $P=0.34$ for femoral neck). Moreover, ANCOVA comparison among treatments showed $P<0.0001$ for the hip and lumbar spine and $P=0.004$ for the femoral neck. ${ }^{45}$ In the AMBER study, ${ }^{46}$ better bone profiles were observed in patients on Drv-Cobi-FTC-Taf vs Drv-CobiFTC-TDF, with a significantly minor decrease in spine and neck BMD. All these observations point to a good safety profile of Drv and Cobi in terms of bone metabolism. Liver toxicity was reported in $0.5 \%$ of patients on treatment with Drv. Due to its pharmacokinetic characteristics (Drv and Cobi are metabolized mainly by the liver) and in consideration of data on hepatic safety, Drv is contraindicated in patients with severe hepatic impairment and severe liver insufficiency. ${ }^{62}$ Central nervous system adverse events have rarely been reported $(<1 \%)$ in randomized clinical trials involving experienced $^{45}$ and naïve patients. ${ }^{46}$

\section{Taf-FTC safety}

Many clinical trials have documented the safety and efficacy of Taf in treatment-naïve and experienced adults in combination with other FDCs. ${ }^{5,6,63}$ These studies observed improvements in median changes of estimated eGFR at week 48 in the switch groups. Median urine protein at week 48 decreased more in the switch groups compared to the control group. No cases of proximal renal tubulopathy or Fanconi syndrome were observed. Fasting lipid values increased from baseline in the switch groups, with no statistically significant changes in total cholesterol:HDL ratio. ${ }^{5,6,63} \mathrm{BMD}$ measured by dual-energy X-ray absorptiometry increased in the switch groups and remained stable or decreased in the control groups, showing a good bone safety profile for Taf compared to TDF.
An interesting meta-analysis ${ }^{64}$ can summarize the aforementioned observations. It identified not only significantly smaller reductions in eGFR-Crocroft-Gault (CG), smaller changes in Retinol Binding Protein (RBP):creatinine $(\mathrm{Cr})$ and urine $\beta_{2}$ microglobulin: $\mathrm{Cr}$ ratio, and less reduction in spine and hip BMD in treatment-naïve patients but also significant efficacy advantages of improved renal function and BMD. These included significant decreases in urine albumin:Cr, urine protein:Cr, urine $\mathrm{RBP}: \mathrm{Cr}$, and urine $\beta_{2}$ microglobulin:Cr ratios and increases in hip and spine BMD by $1.47 \%$ and $1.56 \%$, respectively, compared with continued TDF regimens. Moreover, in this analysis a higher viral suppression rate in patients switching to Taf was observed. ${ }^{64}$ This observation was recently reinforced in a randomized, active-controlled, multicenter, open-label trial that confirmed the superiority of Taf vs TDF in virological efficacy. ${ }^{7}$ Finally, a recent study remarked that since coadministration with Cobi results in significantly higher Tfv concentrations and higher TDF discontinuation compared to other antiretroviral regimens, registration trials of $\mathrm{Taf}^{65}$ may have been prone to bias when evaluating safety comparisons with Taf.

\section{Conclusion}

Drv-Cobi-FTC-Taf is a drug regimen with demonstrated efficacy in HIV infection. The safety and tolerability of this combination is comparable to other STRs available at this time. PIs maintain a role in treatment of HIV infection, especially in naïve patients when an ART regimen needs to be initiated as soon as possible and resistance tests and HLA-B5701 are not available, as well as in experienced patients, when virological failure to nonnucleoside reversetranscriptase inhibitors or integrase strand-transfer inhibitor is demonstrated and an STR with a high genetic barrier and low-toxicity profile is required.

\section{Disclosure}

NS has received grants for board membership by Janssen and ViiV, payment for lectures, including service on speakers' bureaux from MSD, and travel grant/supports from Gilead. AG has received grants/research support from AbbVie, Astellas, BMS, Boehringer, Gilead, Janssen, MSD, Novartis, Pfizer, Roche, ViiV, and ANLAIDS Sezione Lombarda, receipt of honoraria or consultation fees from Bristol-Myers Squibb, Gilead, Janssen, MSD, Novartis, and ViiV, fees for participation in a company-sponsored speakers' bureau from Gilead, and travel grant/supports from Bristol-Myers Squibb, Gilead, Jansen, and ViiV. AB has received grants for board membership from Janssen, grants/research support from 
Gilead and ANLAIDS Sezione Lombarda, and travel grants/ support from ViiV, Gilead, AbbVie, MSD, and BMS. The authors report no other conflicts of interest in this work.

\section{References}

1. Palella FJ, Delaney KM, Moorman AC, et al. Declining morbidity and mortality among patients with advanced human immunodeficiency virus infection. HIV Outpatient Study Investigators. $N$ Engl J Med. 1998;338(13):853-860.

2. Grinspoon S, Carr A. Cardiovascular risk and body-fat abnormalities in HIV-infected adults. N Engl J Med. 2005;352(1):48-62.

3. Molina JM, Andrade-Villanueva J, Echevarria J, et al; CASTLE Study Team. Once-daily atazanavir/ritonavir compared with twice-daily lopinavir/ritonavir, each in combination with tenofovir and emtricitabine, for management of antiretroviral-naïve HIV-1-infected patients: 96-week efficacy and safety results of the CASTLE study. J Acquir Immune Defic Syndr. 2010;53(3):323-332.

4. Orkin C, Dejesus E, Khanlou H, et al. Final 192-week efficacy and safety of once-daily darunavir/ritonavir compared with lopinavir/ritonavir in HIV-1-infected treatment-naïve patients in the ARTEMIS trial. HIV Med. 2013;14(1):49-59.

5. Mills A, Arribas JR, Andrade-Villanueva J, et al; GS-US-292-0109 team. Switching from tenofovir disoproxil fumarate to tenofovir alafenamide in antiretroviral regimens for virologically suppressed adults with HIV-1 infection: a randomised, active-controlled, multicentre, open-label, phase 3, non-inferiority study. Lancet Infect Dis. 2016;16(1):43-52.

6. Pozniak A, Arribas JR, Gathe J, et al; GS-US-292-0112 Study Team. Switching to Tenofovir Alafenamide, Coformulated With Elvitegravir, Cobicistat, and Emtricitabine, in HIV-Infected Patients With Renal Impairment: 48-Week Results From a Single-Arm, Multicenter, Open-Label Phase 3 Study. J Acquir Immune Defic Syndr. 2016;71(5):530-537.

7. Dejesus E, Haas B, Segal-Maurer S, et al. Superior Efficacy and Improved Renal and Bone Safety After Switching from a Tenofovir Disoproxil Fumarate- to a Tenofovir Alafenamide-Based Regimen Through 96 Weeks of Treatment. AIDS Res Hum Retroviruses. 2018;34(4):337-342.

8. Hemmige V, Flash CA, Carter J, Giordano TP, Zerai T. Single tablet HIV regimens facilitate virologic suppression and retention in care among treatment naïve patients. AIDS Care. 2018;30(8):1017-1024.

9. Ray AS, Fordyce MW, Hitchcock MJ. Tenofovir alafenamide: A novel prodrug of tenofovir for the treatment of Human Immunodeficiency Virus. Antiviral Res. 2016;125:63-70.

10. Ogawa E, Furusyo N, Nguyen MH. Tenofovir alafenamide in the treatment of chronic hepatitis B: design, development, and place in therapy. Drug Des Devel Ther. 2017;11:3197-3204.

11. Agarwal K, Fung SK, Nguyen TT, et al. Twenty-eight day safety, antiviral activity, and pharmacokinetics of tenofovir alafenamide for treatment of chronic hepatitis B infection. J Hepatol. 2015;62(3):533-540.

12. Lee WA, He GX, Eisenberg E, et al. Selective intracellular activation of a novel prodrug of the human immunodeficiency virus reverse transcriptase inhibitor tenofovir leads to preferential distribution and accumulation in lymphatic tissue. Antimicrob Agents Chemother. 2005;49(5):1898-1906.

13. Murakami E, Wang T, Park Y, et al. Implications of efficient hepatic delivery by tenofovir alafenamide (GS-7340) for hepatitis B virus therapy. Antimicrob Agents Chemother. 2015;59(6):3563-3569.

14. Custodio JM, Fordyce M, Garner W, et al. Pharmacokinetics and Safety of Tenofovir Alafenamide in HIV-Uninfected Subjects with Severe Renal Impairment. Antimicrob Agents Chemother. 2016;60(9):5135-5140.

15. Custodio JM, Chuck SK, Chu H, et al. Lack of clinically important PK interaction between coformulated ledipasvir/sofosbuvir and rilpivirine/emtricitabine/tenofovir alafenamide. Pharmacol Res Perspect. 2017;5(5):e00353.

16. Rittweger M, Arastéh K. Clinical pharmacokinetics of darunavir. Clin Pharmacokinet. 2007;46(9):739-756.

17. Hull MW, Montaner JS. Ritonavir-boosted protease inhibitors in HIV therapy. Ann Med. 2011;43(5):375-388.
18. Xu L, Liu H, Murray BP, et al. Cobicistat (GS-9350): A Potent and Selective Inhibitor of Human CYP3A as a Novel Pharmacoenhancer. ACS Med Chem Lett. 2010;1(5):209-213.

19. de Nicolò A, Simiele M, Calcagno A, et al. Intracellular antiviral activity of low-dose ritonavir in boosted protease inhibitor regimens. Antimicrob Agents Chemother. 2014;58(7):4042-4047.

20. Navarro J, Curran A. Profile of once-daily darunavir/cobicistat fixeddose combination for the treatment of HIV/AIDS. Hiv Aids. 2016;8: 175-182.

21. Rezolsta [prescribing information]. Available from: http://www.ema. europa.eu/docs/en_GB/document_library/EPAR_-_Product_Information/human/002819/WC500178953.pdf. Accessed March 3, 2018.

22. Kakuda TN, van de Casteele T, Petrovic R, et al. Bioequivalence of a darunavir/cobicistat fixed-dose combination tablet versus single agents and food effect in healthy volunteers. Antivir Ther. 2014;19(6):597-606.

23. Tybost [prescribing information]. Available from: http://www.ema. europa.eu/docs/en_GB/document_library/EPAR_-_Product_Information/human/002572/WC500153014.pdf. Accessed March 3, 2018.

24. Mathias AA, German P, Murray BP, et al. Pharmacokinetics and pharmacodynamics of GS-9350: a novel pharmacokinetic enhancer without anti-HIV activity. Clin Pharmacol Ther. 2010;87(3):322-329.

25. Kakuda TN, Opsomer M, Timmers M, et al. Pharmacokinetics of darunavir in fixed-dose combination with cobicistat compared with coadministration of darunavir and ritonavir as single agents in healthy volunteers. J Clin Pharmacol. 2014;54(8):949-957.

26. Tashima K, Crofoot G, Tomaka FL, et al. Cobicistat-boosted darunavir in HIV-1-infected adults: week 48 results of a Phase IIIb, open-label single-arm trial. AIDS Res Ther. 2014;11:39.

27. Curran A, Pérez-Valero I, Moltó J, Rezolsta MJ. Rezolsta (Darunavir/ Cobicistat): First Boosted Protease Inhibitor Co-formulated with Cobicistat. AIDS Rev. 2015;17(2):114-120.

28. Clay PG, Nag S, Graham CM, Narayanan S. Meta-Analysis of Studies Comparing Single and Multi-Tablet Fixed Dose Combination HIV Treatment Regimens. Medicine. 2015;94(42):e1677.

29. Elliot ER, Amara A, Pagani N, et al. Once-daily atazanavir/cobicistat and darunavir/cobicistat exposure over $72 \mathrm{~h}$ post-dose in plasma, urine and saliva: contribution to drug pharmacokinetic knowledge. J Antimicrob Chemother. 2017;72(7):2143.

30. Bartels H, Decosterd L, Battegay M, Marzolini C. Darunavir concentrations in CSF of HIV-infected individuals when boosted with cobicistat versus ritonavir. J Antimicrob Chemother. 2017;72(9):2574-2577.

31. Marzolini C, Gibbons S, Khoo S, Back D. Cobicistat versus ritonavir boosting and differences in the drug-drug interaction profiles with comedications. J Antimicrob Chemother. 2016;71(7):1755-1758.

32. Nathan B, Bayley J, Waters L, Post FA. Cobicistat: a Novel Pharmacoenhancer for Co-Formulation with HIV Protease and Integrase Inhibitors. Infect Dis Ther. 2013;2(2):111-122.

33. Lepist EI, Phan TK, Roy A, et al. Cobicistat boosts the intestinal absorption of transport substrates, including HIV protease inhibitors and GS-7340, in vitro. Antimicrob Agents Chemother. 2012;56(10):5409-5413.

34. HIV drug Interactions [Homepage on the internet]. University of Liverpool [updated July 4 July 2018 , cited Augyst 3, 2018]. Available from: https:// www.hiv-druginteractions.org/checker. Accessed September 25, 2018.

35. Tseng A, Hughes CA, Wu J, Seet J, Phillips EJ. Cobicistat Versus Ritonavir: Similar Pharmacokinetic Enhancers But Some Important Differences. Ann Pharmacother. 2017;51(11):1008-1022.

36. Roberts O, Khoo S, Owen A, Siccardi M. Interaction of Rifampin and Darunavir-Ritonavir or Darunavir-Cobicistat In Vitro. Antimicrob Agents Chemother. 2017;61(5).

37. Tseng AL, Luetkehoelter J, Walmsley SL. Increase in international normalized ratio after switching from atazanavir/ritonavir to darunavir/ cobicistat in a patient on warfarin: boosters are not always equal. AIDS. 2017;31(1):175-176.

38. Ramanathan S, Wang H, Szwarcberg J, Kearney BP. Safety/tolerability, pharmacokinetics and boosting of twice-daily cobicistat administered alone or in combination with darunavir or tipranavir. 13th International Workshop on Clinical Pharmacology of HIV Therapy; April 16-18, 2012; Barcelona, Spain. 
39. Clinicaltrials.gov [Homepage on the internet]. U.S. National Library of Medicine [updated september 24 2018, cited August 3, 2018] Available from: https://clinicaltrials.gov/ct2/show/NCT02475135.\%20 Accessed\%2025\%20September\%2025" https://clinicaltrials.gov/ct2/ show/NCT02475135. Accessed September 25, 2018.

40. Crauwels H, Baugh B, van Landuyt E, Vanveggel S, Hijzen A, Opsomer M. Impact of food on the bioavailability of darunavir, cobicistat, emtricitabine and tenofovir alafenamide (DCFTAF), the first protease inhibitor-based complete HIV-1 regimen HIV Drug Therapy 23-26 October, 2016; Glasgow.

41. Clinicaltrials. gov [Homepage on the internet]. U.S. National Library of Medicine [updated september 24 2018, cited August 3, 2018] Available from https://clinicaltrials.gov/ct2/show/NCT01565850. Accessed September 25, 2018.

42. Mills A, Crofoot G, Mcdonald C, et al. Tenofovir Alafenamide Versus Tenofovir Disoproxil Fumarate in the First Protease Inhibitor-Based Single-Tablet Regimen for Initial HIV-1 Therapy: A Randomized Phase 2 Study. J Acquir Immune Defic Syndr. 2015;69(4):439-445.

43. Clinicaltrials. gov [Homepage on the internet]. U.S. National Library of Medicine [updated september 24 2018, cited August 3, 2018]. Available from: https://clinicaltrials.gov/ct2/show/NCT02269917. Accessed September 25, 2018.

44. Clinicaltrials.gov [Homepage on the internet]. U.S. National Library of Medicine [updated september 24 2018, cited August 3, 2018]. Available from: https://clinicaltrials.gov/ct2/show/NCT02431247. Accessed September 25, 2018.

45. Orkin C, Molina JM, Negredo E, et al; EMERALD study group. Efficacy and safety of switching from boosted protease inhibitors plus emtricitabine and tenofovir disoproxil fumarate regimens to single-tablet darunavir, cobicistat, emtricitabine, and tenofovir alafenamide at 48 weeks in adults with virologically suppressed HIV-1 (EMERALD): a phase 3, randomised, non-inferiority trial. Lancet HIV. 2018;5(1):e23-e34

46. Eron J, Orkin C, Gallant J. Week 48 results of AMBER: A Phase 3, randomised, double-blind trial in antiretroviral treatment-naïve HIV1-infected adults to evaluate the efficacy and safety of the once-daily, single-tablet regimen of darunavir/cobicistat/emtricitabine/tenofovir alafenamide $(\mathrm{D} / \mathrm{C} / \mathrm{F} / \mathrm{TAF})$ versus darunavir/cobicistat plus emtricitabine/tenofovir disoproxil fumarate. EACS 2017; October 25-27, 2017; Milan, Italy.

47. HIV Drug Resistance Database [Homepage on the internet]. Stanford University [updated July 18 2018, cited August 3, 2018] Available from: https://hivdb.stanford.edu/hivdb/by-mutations/. Accessed September 25, 2018.

48. German P, Liu HC, Szwarcberg J, et al. Effect of cobicistat on glomerular filtration rate in subjects with normal and impaired renal function. $J$ Acquir Immune Defic Syndr. 2012;61(1):32-40.

49. Stray KM, Bam RA, Birkus G, et al. Evaluation of the effect of cobicistat on the in vitro renal transport and cytotoxicity potential of tenofovir. Antimicrob Agents Chemother. 2013;57(10):4982-4989.

50. Krutzén E, Bäck SE, Nilsson-Ehle I, Nilsson-Ehle P. Plasma clearance of a new contrast agent, iohexol: a method for the assessment of glomerular filtration rate. J Lab Clin Med. 1984;104(6):955-961.

51. Fisher M, Mcdonald C, Moyle G, et al. Switching from ritonavir to cobicistat in HIV patients with renal impairment who are virologically suppressed on a protease inhibitor. J Int AIDS Soc. 2014;17(4 Suppl 3): 19824.
52. Tungsiripat M, Kitch D, Glesby MJ, et al. A pilot study to determine the impact on dyslipidemia of adding tenofovir to stable background antiretroviral therapy: ACTG 5206. AIDS. 2010;24(11):1781-1784.

53. Auclair M, Afonso P, Capel E, Caron-Debarle M, Capeau J. Impact of darunavir, atazanavir and lopinavir boosted with ritonavir on cultured human endothelial cells: beneficial effect of pravastatin. Antivir Ther. 2014;19(8):773-782.

54. Guaraldi G, Zona S, Cossarizza A, et al. Randomized trial to evaluate cardiometabolic and endothelial function in patients with plasma HIV-1 RNA suppression switching to darunavir/ritonavir with or without nucleoside analogues. HIV Clin Trials. 2013;14(4):140-148.

55. Ryom L, Lundgren JD, et al. D:A:D study group, et al. Cardiovascular disease and use of contemporary protease inhibitors: the D:A:D international prospective multicohort study. Lancet HIV. 2018;5(6): e291-e300.

56. Opsomer M, Dimitrova D, Verspeelt J. Evaluation of Cardiovascular Disease (CVD) Risk in HIV-1-infected Patients Treated With Darunavir/Ritonavir (DRV/r). 11th Annual American Conference for the Treatment of HIV (ACTHIV); April 20-22, 2017; Dallas, Texas.

57. Bernardino JI, Mocroft A, Mallon PW, et al; NEAT001/ANRS143 Study Group. Bone mineral density and inflammatory and bone biomarkers after darunavir-ritonavir combined with either raltegravir or tenofovir-emtricitabine in antiretroviral-naïve adults with HIV-1: a substudy of the NEAT001/ ANRS143 randomised trial. Lancet HIV. 2015;2(11):e464 e473.

58. Raffi F, Babiker AG, Richert L, et al; NEAT001/ANRS143 Study Group. Ritonavir-boosted darunavir combined with raltegravir or tenofovir-emtricitabine in antiretroviral-naïve adults infected with HIV-1: 96 week results from the NEAT001/ANRS143 randomised non-inferiority trial. Lancet. 2014;384(9958):1942-1951.

59. Kearney BP, Mathias A, Mittan A, Sayre J, Ebrahimi R, Cheng AK Pharmacokinetics and safety of tenofovir disoproxil fumarate on coadministration with lopinavir/ritonavir. J Acquir Immune Defic Syndr. 2006;43(3):278-283.

60. Brown TT, Moser C, Currier JS, et al. Changes in Bone Mineral Density After Initiation of Antiretroviral Treatment With Tenofovir Disoproxil Fumarate/Emtricitabine Plus Atazanavir/Ritonavir, Darunavir/Ritonavir, or Raltegravir. J Infect Dis. 2015;212(8):1241-1249.

61. Mccomsey GA, Kitch D, Daar ES, et al. Bone mineral density and fractures in antiretroviral-naïve persons randomized to receive abacavirlamivudine or tenofovir disoproxil fumarate-emtricitabine along with efavirenz or atazanavir-ritonavir: Aids Clinical Trials Group A5224s, a substudy of ACTG A5202. J Infect Dis. 2011;203(12):1791-1801.

62. Capetti A, Cossu MV, Rizzardini G. Darunavir/cobicistat for the treatment of HIV-1: a new era for compact drugs with high genetic barrier to resistance. Expert Opin Pharmacother. 2015;16(17):2689-2702.

63. Wohl D, Oka S, Clumeck N, et al; GS-US-2,92-01040111 and Study Team. Brief Report: A Randomized, Double-Blind Comparison of Tenofovir Alafenamide Versus Tenofovir Disoproxil Fumarate, Each Coformulated With Elvitegravir, Cobicistat, and Emtricitabine for Initial HIV-1 Treatment: Week 96 Results. J Acquir Immune Defic Syndr. 2016;72(1):58-64.

64. Wang H, Lu X, Yang X, Xu N. The efficacy and safety of tenofovir alafenamide versus tenofovir disoproxil fumarate in antiretroviral regimens for HIV-1 therapy: Meta-analysis. Medicine. 2016;95(41):e5146.

65. Cattaneo D, Minisci D, Baldelli S, et al. Effect of Cobicistat on Tenofovir Disoproxil Fumarate (TDF): What Is True for TAF May Also Be True for TDF. J Acquir Immune Defic Syndr. 2018;77(1):86-92.

\section{Publish your work in this journal}

Drug Design, Development and Therapy is an international, peerreviewed open-access journal that spans the spectrum of drug design and development through to clinical applications. Clinical outcomes, patient safety, and programs for the development and effective, safe, and sustained use of medicines are the features of the journal, which

\section{Dovepress}

has also been accepted for indexing on PubMed Central. The manuscript management system is completely online and includes a very quick and fair peer-review system, which is all easy to use. Visit http://www.dovepress.com/testimonials.php to read real quotes from published authors. 